\title{
Bioestimulantes comerciais na germinação de sementes de sorgo granífero
}

\author{
Vinícius de Carvalho', Josef Gastl Filho², Millena Almeida Resende³, Muriel Silva Vilarinho', Sávio Luiz de Santi', \\ Victor Prissinotto Marques' \\ I Universidade do Estado de Minas Gerais (UEMG), Unidade Ituiutaba, Ituiutaba, MG. \\ E-mail: viniciuscarvalho098@gmail.com, muriel.vilarinho@uemg.br, saviodesanti@gmail.com, vitor-302009@hotmail.com \\ 2 Instituto de Ciências Agrárias Universidade Federal de Uberlândia (UFU), Campus Glória, Uberlândia, MG. \\ E-mail: josef.gastl@ufu.br \\ ${ }^{3}$ Instituto de Biotecnologia, Universidade Federal de Uberlândia (UFU), Campus Umuarama, Uberlândia, MG. \\ E-mail: millenaresende19@ufu.br
}

\section{Resumo}

São várias as tecnologias aplicadas visando atingir o potencial genético das culturas agrícolas, dentre elas estão os bioestimulantes que se destacam por agirem diretamente na germinação e no desenvolvimento das plântulas influenciando a produtividade agrícola. Neste sentido, o presente estudo teve por objetivo comparar o efeito da dosagem técnica de diferentes bioestimulantes comerciais na germinação do sorgo granífero. $O$ experimento foi conduzido em delineamento inteiramente casualizado, com 5 tratamentos $\left(8,0 \mathrm{~mL}\right.$ de Awaken ${ }^{\circledR} \mathrm{kg}^{-1}$ de semente; 0, I4 mL de Stimulate ${ }^{\circledR} \mathrm{L}^{-1} ; 0,0$ I4 $\mathrm{mL}$ de $\operatorname{Vorax}^{\circledR} \mathrm{L}^{-1}$; $6,0 \mathrm{~mL}$ de Zc Full Patriot ${ }^{\circledR} \mathrm{kg}^{-1}$ de sementes e testemunha) e 4 repetições de 50 sementes. As sementes foram semeadas em rolos de papel Germitest e acondicionadas em germinador a $25^{\circ} \mathrm{C}$ por 10 dias. Os caracteres avaliados foram primeira contagem, índice de velocidade de germinação, porcentagem de germinação, comprimento de plântula e biomassas fresca e seca de plântulas. Não foram verificadas diferenças significativas para a primeira contagem, biomassa fresca e índice de velocidade de germinação. Os bioestimulantes afetaram positivamente o comprimento de plântula em relação à testemunha, sendo os maiores valores conferidos por Vorax ${ }^{\circledR}$ e Zc Full Patriot ${ }^{\circledR}$. Com exceção do Awaken ${ }^{\circledR}$ que apresentou o menor valor, todos os tratamentos apresentaram altos percentuais de germinação. $\bigcirc$ Stimulate ${ }^{\circledR}$ e Awaken ${ }^{\circledR}$ conferiram maior acúmulo de biomassa seca, em detrimento do Vorax ${ }^{\circledR}$ e do Zc Full Patriot ${ }^{\circledR}$. Conclui-se que a dosagem do Awaken ${ }^{\circledR}$ afetou negativamente a maioria dos caracteres, enquanto que a dosagem aplicada do Stimulate ${ }^{\circledR}$ conferiu bom desempenho em todos dos caracteres.

Palavras-chave: Biorregulador. Micronutriente. Regulador Vegetal. Sorghum Bicolor. Vigor.

\section{Abstract \\ Commercial bioestimulants on the germination of grain sorghum seeds}

Among the various technologies that are applied in order to achieve the genetic potential of agricultural crops are the biostimulants that stand out for acting directly on germination and seedling development influencing agricultural productivity. In this sense, the present study aimed to compare the effect of the technical dosage of different commercial biostimulants on the germination of grain sorghum. The experiment was conducted in a completely randomized design, with 5 treatments $\left(8.0 \mathrm{~mL}\right.$ Awaken ${ }^{\circledR} \mathrm{kg}^{-1}$ seed; $0.14 \mathrm{~mL}$ Stimulate ${ }^{\circledR} \mathrm{L}^{-1} ; 0.014 \mathrm{~mL}$ Vorax ${ }^{\circledR} \mathrm{L}^{-1} ; 6.0 \mathrm{~mL}$ Zc Full Patriot ${ }^{\circledR}$ $\mathrm{kg}^{-1}$ seed and control) and 4 repetitions of 50 seeds. The seeds were sown onto germitest paper roll and incubated in a germination chamber at $25^{\circ} \mathrm{C}$ for 10 days. The evaluated characters were first germination count, germination speed index, germination percentage, seedling length and fresh and dried biomass of seedlings. There were no significant differences for the first germination count, for the fresh biomass and germination speed index. All the biostimulants 
positively influenced the seedling length in relation to the control and the highest values were given by Vorax ${ }^{\circledR}$ and Zc Full Patriot ${ }^{\circledR}$. With the exception of Awaken ${ }^{\circledR}$, which presented the lowest value, all treatments had high germination percentages. Stimulate ${ }^{\circledR}$ and Awaken ${ }^{\circledR}$ conferred greater accumulation of dry biomass, to the detriment of Vorax ${ }^{\circledR}$ and Zc Full Patriot ${ }^{\circledR}$. It is concluded that the Awaken ${ }^{\circledR}$ dosage negatively affected most of the characters, while the applied Stimulate ${ }^{\circledR}$ dosage resulted in good performance in all of the characters.

Keywords: Bioregulator. Micronutrient. Plant Growth Regulators. Sorghum Bicolor. Vigor.

\section{Introdução}

Originário do centro leste da África e parte da Ásia, o sorgo é uma planta pertencente à família Poaceae, do gênero Sorghum e da espécie Sorghum bicolor (L.) Moench (ROCHA, 2017). É uma espécie diploide com $2 n=20$ cromossomos (ALMEIDA FILHO, 20I2), gramínea anual, autógama, com baixa taxa de fecundação cruzada (RODRIGUES et al., 2009), planta com metabolismo C4, ou seja, gera como primeiro produto da fotossíntese um composto de 4 carbonos, sendo mais eficiente quanto a utilização de água pela regulação da abertura dos estômatos, o que proporciona maior tolerância a elevados níveis de radiação solar (CUNHA; SEVERO FILHO, 2010).

É uma cultura muito versátil e socioeconomicamente importante, utilizada para produção de grãos, de feno, de silagem e pastejo direto (SANTOS, 20I I). A área cultivada de sorgo no Brasil na safra 20I9/20 foi de 808,7 mil hectares, com uma produção de grãos de 2,63 I milhões toneladas e produtividade média de 3,254 toneladas ha-1 (CONAB, 2020). Essa elevada difusão se deve à grande adaptabilidade aos mais diferentes tipos de ambientes, pois, apresenta tolerância à seca e às limitações de nutrientes no solo, sendo boa opção em regiões impróprias para o plantio do milho (SANTOS, 20I I).

Em função da criação do grupo Pró-sorgo no início dos anos 90, do crescente uso da cultura nos sistemas de plantio direto nas regiões Centro-Oeste e Sudeste, onde o sorgo começou a ser utilizado como sendo uma opção para rotação de cultura nas áreas de agricultura com monocultivo, e o crescente uso na safrinha em função das condições edafoclimáticas regionais na região central do Brasil, proporcionaram um crescimento significativo da cultura (PARRELLA et al., 2010).

Nos últimos anos têm crescido a utilização de produtos que favorecem a expressão do potencial genético das plantas mediante alterações dos processos vitais e estruturais, promovendo um equilíbrio hormonal e estimulando o desenvolvimento do sistema radicular (SILVA et al., 2008). Segundo Kolling et al. (20 I6) estes produtos consistem dos bioestimulantes, que são definidos como sendo uma mistura de reguladores de crescimento vegetal ou de um ou mais reguladores vegetais com outros compostos de natureza bioquímica diferente (aminoácidos, nutrientes, vitaminas, etc).

Gastl Filho et al. (2017) afirmaram que o uso de reguladores vegetais e nutrientes que apresentam ação direta na germinação de sementes e no desenvolvimento das plântulas tem potencializado o aumento da produtividade em culturas como algodão, arroz, feijão, milho e soja, embora não seja prática rotineira entre produtores de culturas de alto nível tecnológico.

Ferreira et al. (2019) verificaram que o intervalo de 5,5 a 6,0 mL de bioestimulante Matriz G (50 mg L'-1 de auxina; $100 \mathrm{mg} \mathrm{L}^{-1}$ de citocinina e $50 \mathrm{mg} \mathrm{L}^{-1}$ de giberelina) associado à adubação nitrogenada melhorou significativamente o crescimento e o rendimento das plantas de sorgo. Entretanto, no estudo de Vendruscolo et al. (20l4) não foi observada influência da aplicação de doses de substâncias húmicas na germinação e desenvolvimento inicial de plântulas de sorgo.

Alguns exemplos de bioestimulantes comerciais existentes no mercado são o Awaken ${ }^{\circledR}(16,00 \%$ de $\mathrm{N}$; 3,53\% de Zn; 0,02\% de B; 0, I5\% de Mn; 0, I5\% de Cu; 0, I5\% de Fe), o Stimulate ${ }^{\circledR}\left(50,0 \mathrm{mg} \mathrm{L}^{-1}\right.$ de ácido giberélico, $50,0 \mathrm{mg} \mathrm{L}^{-1}$ de ácido 4-indol-3-ilbutírico e $90,0 \mathrm{mg} \mathrm{L}^{-1}$ de cinetina), o Vorax ${ }^{\circledR}$ [4\% de nitrogênio (50 $\mathrm{g} \mathrm{L}^{-1}$ de N), I8\% de carbono orgânico total (225 g L-1 de COT), 25\% de Aminoácido Ácido L-Glutâmico, I\% Extrato de Alga e 0,5\% de Tensoativo] e o Zc Full Patriot ${ }^{\circledR}$ (N, K, B, Cu, Fe, Mn e Zn).

Apesar das vantagens apresentadas, os resultados dos trabalhos de pesquisa com o tratamento de sementes com bioestimulantes são contraditórios. Neste sentido, o presente estudo teve por objetivo comparar a dose recomendada de bioestimulantes comerciais na germinação do sorgo granífero. 


\section{Material e Métodos}

O presente estudo foi conduzido no Laboratório de Análise de Sementes da Universidade do Estado de Minas Gerais (UEMG), Unidade Ituiutaba, no município de Ituiutaba (MG), pelo período de maio a junho de 2019. A cultivar de sorgo utilizada foi a IGI00 da empresa Brevant Sementes ${ }^{\circledR}$, safra 2018/20I9.

O experimento foi conduzido em delineamento inteiramente casualizado, com 5 tratamentos (Testemunha; Awaken ${ }^{\circledR}$; Stimulate ${ }^{\circledR}$; Vorax ${ }^{\circledR}$ e Zc Full Patriot ${ }^{\circledR}$ ) e 4 repetições de 50 sementes (BRASIL, 2009). Os tratamentos consistiram da aplicação de bioestimulantes conforme a dose recomendada pelo fabricante. Os bioestimulantes Stimulate ${ }^{\circledR}$ e Vorax ${ }^{\circledR}$ não possuem recomendação para a cultura do sorgo, sendo assim adotada a dose aplicada na cultura do milho, tendo em vista a proximidade morfogenéticas entre as duas culturas. As dosagens aplicadas foram: $8,0 \mathrm{~mL}$ de Awaken ${ }^{\circledR} \mathrm{kg}^{-1}$ de semente; 0, I4 mL de Stimulate ${ }^{\circledR} \mathrm{L}^{-1} ; 0,0$ I4 $\mathrm{mL}$ de Vorax ${ }^{\circledR} \mathrm{L}^{-1} ; 6,0 \mathrm{~mL}$ de Zc Full Patriot ${ }^{\circledR} \mathrm{kg}^{-1}$ de sementes.

Conforme metodologia preconizada pelas Regras para Análise de Sementes, para o teste de germinação as sementes foram colocadas sobre duas folhas de papel Germitest, cobrindo-as com outra folha, sendo, em seguida umedecidas com volume de 2,5 vezes a massa das 3 folhas secas, com os respectivos tratamentos. Posteriormente, foram confeccionados rolos, que foram acondicionados em germinador à temperatura de $25^{\circ} \mathrm{C}$, pelo período de 10 dias (BRASIL, 2009). O processo de germinação foi monitorado diariamente, com a contagem de plântulas normais do $4^{\circ}$ ao $7^{\circ}$ dia. Para a semente ser considerada germinada o critério adotado foi a radícula possuir $2 \mathrm{~mm}$ ou mais.

As características avaliadas no teste de germinação foram: primeira contagem (PC) no quarto dia (BRASIL, 2009), índice de velocidade de germinação (IVG) (MAGUIRE, 1962) e porcentagem de germinação (\%G) (NASSIF; PEREZ, 2000). As equações de IVG (I) e \%G (2) utilizadas foram:

$$
I V G=\sum \frac{n_{i}}{t_{i}}(1)
$$

Em que: ni = número de sementes germinadas por dia; ti = tempo de incubação (dias).

$$
\% G=\frac{A}{N} \times 100(2)
$$

Em que: \%G = porcentagem de germinação; $N=$ número de sementes germinadas; $A=$ número total de sementes colocadas para germinar.

Ao final do período de germinação as plântulas normais foram avaliadas quanto ao comprimento de plântula (CP), utilizando-se uma régua milimetrada, sendo o resultado final expresso em $\mathrm{cm}_{\text {planta }}{ }^{-1}$. A biomassa fresca de plântulas (BFP) consistiu na pesagem da parcela experimental, em uma balança analítica com precisão de $0,000 \mathrm{lg}$, em seguida, as plântulas foram acondicionadas em cápsulas metálicas e colocadas para secar em estufa a $\pm 105^{\circ} \mathrm{C}$ até peso constante para determinação da biomassa seca de plântula (BSP), os resultados finais foram expresso em $g$ parcela ${ }^{-1}$.

Após a coleta dos dados, os valores foram submetidos ao teste de Shapiro-Wilk para verificar a normalidade dos resíduos e ao teste de Levene para mediana para verificar a homocedasticidade. Posteriormente, os dados foram submetidos à análise de variância (ANOVA) e aplicados os testes de médias bilaterais de Dunnett $(p>0,05)$, para comparação dos tratamentos em relação à testemunha, e as médias dos tratamentos foram comparadas entre si, pelo teste de comparação múltipla de Scott Knott $(p>0,05)$. O Programa estatístico utilizado foi o R version 3.6.2 (Dark and Stormy Night) (R CORE TEAM, 20I9).

\section{Resultados e Discussões}

A análise de variância pelo teste $F$ demonstrou haver diferenças significativas $(p<0,05)$ para os caracteres de CP, \%G e BSP de sorgo granífero cultivar IGI00 tratadas com diferentes bioestimulantes comerciais (Tabela I).

Para o caractere $\mathrm{CP}$, todos os bioestimulantes se diferiram significativamente da testemunha, tendo apresentado os maiores valores. Os bioestimulantes Vorax ${ }^{\circledR}$ e Zc Full Patriot ${ }^{\circledR}$ proporcionaram CP significativamente maior quando comparado com os demais tratamentos, ou seja, os reguladores vegetais, nutrientes e demais compostos presentes na formulação dos bioestimulantes estimularam a elongação celular das plântulas de sorgo granífero. 
Tabela I - Comprimento de plântula (CP), primeira contagem (PC), porcentagem de germinação (\%G), biomassa fresca de plântulas (BFP), biomassa seca de plântulas (BSP) e índice de velocidade de germinação (IVG) de sementes de sorgo granífero cultivar IGI00 tratadas com diferentes bioestimulantes comerciais.

\begin{tabular}{|c|c|c|c|c|c|c|}
\hline \multirow{2}{*}{ Bioestimulante } & $\mathrm{CP}$ & PC & $\% G$ & BFP & BSP & \multirow{2}{*}{ IVG } \\
\hline & $\mathrm{cm}_{\text {plântula }}{ }^{-1}$ & \multicolumn{2}{|c|}{$\%$} & \multicolumn{2}{|c|}{ g parcela-1 } & \\
\hline Testemunha & $2,9050 \mathrm{c}$ & 47,0 & $95,0 \mathrm{a}$ & 4,1550 & I,2075 a & 10,2667 \\
\hline Awaken ${ }^{\circledR}$ & *3,7075 b & $* 18,5$ & *88,5 b & 4,7543 & $I, 4160 \mathrm{a}$ & * 8,7792 \\
\hline Stimulate ${ }^{\circledR}$ & *3,5475 b & 31,5 & $94,0 \mathrm{a}$ & 4,7663 & I,2728 a & 9,9208 \\
\hline Vorax $^{\circledR}$ & $* 4,0375 \mathrm{a}$ & 21,5 & $95,0 \mathrm{a}$ & 4,8945 & *0,9385 b & 9,5625 \\
\hline Zc Full Patriot ${ }^{\circledR}$ & $* 4,0550 \mathrm{a}$ & 28,5 & $92,5 \mathrm{a}$ & 4,9130 & $* 0,7748$ b & 9,3958 \\
\hline Teste de F & **** & ns & 米米 & ns & ***** & **⿻丷木 \\
\hline CV (\%) & 7,49 & 49,87 & 2,32 & 8,78 & 10,68 & 5,65 \\
\hline Normalidade' & SIM & SIM & SIM & SIM & SIM & SIM \\
\hline Homogeneidade 2 & SIM & SIM & SIM & SIM & SIM & SIM \\
\hline
\end{tabular}

Médias seguidas por letras distintas nas colunas se diferem pelo teste de Scott Knott $(p<0,05)$. *Tratamento difere da testemunha pelo teste de Dunnet $(p<0,05)$. ** Significativo pelo teste de $\mathrm{F}(p<0,0 \mathrm{I})$. **** Significativo pelo teste de $\mathrm{F}(p<0,00 \mathrm{I})$. ns $\mathrm{Não}$ significativo. 'Normalidade dos resíduos pelo teste de Shapiro-Wilk, em nível de $5 \%$ de probabilidade de erro. ${ }^{2}$ Homogeneidade das variâncias pelo teste de Levene para mediana, em nível de 5\% de probabilidade de erro. Fonte: Ituiutaba, MG, 2019.

Resultados superiores foram obtidos por Vazquez et al. (201 I) avaliando a qualidade fisiológica de diferentes lotes de sementes de sorgo, os quais obtiveram valores de CP superiores a $6,16 \mathrm{~cm}$. Da mesma forma, Cunha et al. (2015) obtiveram valores de II,4 e I2,2 cm para as cultivares de sorgo BRS6I0 e Volumax, respectivamente. Buchelt et al. (2019) afirmam que os reguladores vegetais do grupo das auxinas, citocininas e giberelinas, são responsáveis por promover a divisão, diferenciação e o alongamento celular, o que resulta no maior comprimento total das plântulas tratadas em relação às não tratadas com bioestimulantes.

Não foi verificada diferença significativa entre os tratamentos aplicados quanto ao caractere PC, isto é, nenhum dos bioestimulantes proporcionou melhor ou pior desempenho germinativo das sementes de sorgo. Quando comparado com a testemunha, o bioestimulante Awaken ${ }^{\circledR}$ se diferenciou significativamente, tendo apresentado o menor PC.

Resultados superiores foram obtidos por Vazquez et al. (201I) ao avaliarem a qualidade fisiológica de diferentes lotes de sementes de sorgo, obtendo PC com índices de germinação superiores a 87,0\%. CoeIho et al. (2014) ao avaliarem a PC de germinação das cultivares de sorgo F305, BRS655, BRS6I0, Volumax I.0I5.045 e I.016.005 obtiveram, respectivamente, $91,0 \%, 91,0 \%, 80,0 \%, 88,0 \%, 94,0 \%$ e $76 \%$, resultados superiores ao do presente estudo.

Para o caractere de \%G foram verificadas diferenças significativas entre os tratamentos, sendo que os bioestimulantes Stimulate ${ }^{\circledR}$, Vorax ${ }^{\circledR}$, Zc Full Patriot ${ }^{\circledR}$ e a testemunha apresentaram os maiores índices germinativos, enquanto que o bioestimulante Awaken ${ }^{\circledR}$ proporcionou menor valor de \%G. Além do mais, quando comparado com a testemunha o Awaken ${ }^{\circledR}$ foi significativamente inferior.

Ao avaliarem os efeitos do tratamento de sementes de sorgo com fertilizantes à base de zinco e molibdenênio, Cunha et al. (2015) obtiveram \%G de $86 \%$ para a cultivar BRS610 e $90 \%$ para a cultivar Volumax, resultados similares ao do presente estudo. Do mesmo modo, ao avaliarem a qualidade fisiológica de diferentes lotes de sementes de sorgo, Vazquez et al. (20I I) obtiveram \%G superiores a 88,0\%. Em seu estudo Coelho et al. (20I4) ao avaliarem a \%G das cultivares de sorgo F305, BRS655, BRS6I0, Volumax I.0I5.045 e I.016.005 obtiveram, respectivamente, $93,0 \%, 91,0 \%, 80,0 \%, 88,0 \%, 94,0 \%$ e $76,0 \%$, resultados próximos ao do presente estudo.

Silva et al. (2008) verificaram em seu estudo com Stimulate que a \%G da linhagem de milho L57 foi reduzida em $38 \%$, entretanto para o milho cultivar GNZ 2004, houve um incremento de $29 \%$ na \%G. Em seu estudo, Santos et al. (20l3) também verificaram respostas positivas em relação ao vigor de plântulas e germinação de girassol quando suas sementes foram tratadas com Stimulate ${ }^{\circledR}$.

Buchelt et al. (2019) afirmaram que o uso das giberelinas presentes no Stimulate ${ }^{\circledR}$ melhora o processo de germinação, devido ao fato de as giberelinas estarem relacionadas com a síntese de enzimas hidrolíticas, que degradam as proteínas e o amido, sendo usadas no desenvolvimento do embrião e também no alongamento da radícula. 
Tendo em vista, os resultados obtidos para a PC e a \%G, é possível inferir que os nutrientes que compõem o bioestimulante Awaken ${ }^{\circledR}$ tiveram ação fitotóxica na concentração aplicada nas sementes de sorgo granífero, o que resultou na redução significativa da germinação e vigor, isto é, a qualidade fisiológica das sementes foi afetada, quando comparada a redução ocorreu tanto em relação à testemunha quanto em relação aos demais bioestimulantes estudados.

Em relação ao caractere de BFP não foram verificadas diferenças significativas entre os tratamentos e nem em relação à testemunha. Bontempo et al. (2016) explicaram que o motivo de muitos experimentos não apresentarem resultados expressivos quanto à utilização de bioestimulantes pode estar relacionado com o fato do estudo ter sido conduzido sob condições edafoclimáticas ideais para a cultura, sendo que os efeitos da aplicação de bioestimulantes só é perceptível nas sementes que são submetidas a condições de estresse, seja ele biótico ou abiótico.

Fato constatado por Tatto et al. (20l8) que ao avaliar a germinação de sementes de soja, verificou que as plântulas tiveram melhor desempenho quando tiveram suas sementes tratadas aplicação exógena de bioestimulante, o que resultou na indução à resistência a condições de estresse hídrico.

Resultados superiores foram obtidos por Cunha et al. (2015) ao avaliarem o efeito da aplicação de fertilizantes compostos de zinco e molibdênio em sementes de sorgo, obtiveram para BFP 9,7 e 9,3 g parcela-1 das cultivares BRS 610 e Volumax, respectivamente. Por sua vez, Vazquez et al. (201 I) obtiveram BFP de sorgo variando entre 3,97 a 7,65 g parcela ${ }^{-1}$.

Foram verificadas diferenças significativas entre os tratamentos em relação ao caractere BSP, sendo que a testemunha e os bioestimulantes Awaken ${ }^{\circledR}$ e Stimulate ${ }^{\circledR}$ apresentaram o maior acúmulo de biomassa seca, quando comparado aos bioestimulantes Vorax ${ }^{\circledR}$ e Zc Full Patriot ${ }^{\circledR}$. Estes dois últimos se diferenciaram significativamente da testemunha. Desta maneira, com base nos resultados é possível afirmar que os compostos constituintes dos bioestimulantes Vorax ${ }^{\circledR}$ e Zc Full Patriot ${ }^{\circledR}$ exerceram ação fitotóxica no acúmulo de biomassa seca por parte das plântulas de sorgo granífero, o que sinaliza que a concentração utilizada não é a ideal para a cultura.

Vazquez et al. (20I I) obtiveram, ao avaliar a qualidade fisiológica de sementes de sorgo, BFP variando entre 0,33 a I,23 g parcela ${ }^{-1}$, resultados próximos ao do presente estudo. Por sua vez, Cunha et al. (20I5) ao avaliarem o efeito da aplicação de fertilizantes compostos de zinco e molibdênio em sementes de sorgo, obtiveram para BSP 6,5 e 7,6 g parcela-1 das cultivares BRS 610 e Volumax, respectivamente.

De modo similar ao presente estudo, Silva et al. (2008) verificaram redução no valor de BSP quando as sementes de milho foram tratadas com bioestimulantes, ou seja, não foram constatados efeitos positivos oriundos do uso de bioestimulantes, os mesmos autores afirmam que provavelmente os micronutrientes presentes, tal como o zinco e o molibdênio, podem ter exercido efeito fitotóxico às sementes, tendo em vista que aplicações em concentrações superiores podem interferir no metabolismo e crescimento.

Ao aplicar o bioestimulante $\operatorname{Orgasol}^{\circledR}$, que contém enxofre, boro, manganês, molibdênio e zinco em sua composição, Worma et al. (20l9) verificaram que os tratamentos não diferiram da testemunha, não havendo incremento nos caracteres avaliados, ou seja, os autores inferiram que não houve efeitos fitóxicos do produtos no processo germinativo.

Em relação ao caractere IVG não foram verificadas diferenças significativas entre os tratamentos. Por sua vez, o bioestimulante Awaken ${ }^{\circledR}$ foi o único a se diferenciar significativamente da testemunha quando considerado o IVG, tendo apresentado valor inferior, ou seja, as sementes tiveram seu vigor reduzido mediante a aplicação do Awaken ${ }^{\circledR}$.

Vazquez et al. (20II) ao avaliarem o índice de velocidade de emergência de diferentes lotes de sementes de sorgo, obtiveram valores entre I5,2 a 17,3, valores superiores ao IVG obtido do presente estudo. Diferentemente do presente estudo, Inoue et al. (20l2) verificaram que as sementes de algodoeiro que foram tratadas com o protetor dietholate isolado e associado com o bioestimulante Awaken ${ }^{\circledR}$, apresentou maior porcentagem de emergência e índice de velocidade de emergência.

Segundo Buchelt et al. (2019) os bioestimulantes são capazes de provocar alterações estruturais responsáveis pelo aumento do vigor das sementes, tal como verificado para os caracteres de CP, IVG e BSP do presente estudo. Segundo Cunha et al. (2015) a aplicação de fertilizante composto de zinco e molibdênio influência de forma positiva a qualidade fisiológica de sementes de sorgo. Entretanto, a depender da concentração de bioestimulante utilizada, pode afetar de forma negativa os caracteres germinativos e de vigor 
das plântulas de sorgo, a exemplo do estudo de Lisboa et al. (2017), os quais inferiram que o uso de altas concentrações do regulador vegetal ácido salicílico influenciou negativamente em até $95,0 \%$ os caracteres de IVG, \%G, comprimento da parte aérea e radicular, BFP e BSP de sorgo sacarino.

Worma et al. (2019) utilizaram em seu estudo uma dosagem única para cada bioestimulante, os mesmos autores afirmaram que a dose pode ter sido sub ou superestimada, o que impede a detecção do efeito exato dos produtos. Portanto, é necessário enfatizar que o presente estudo aplicou as dosagens técnicas recomendadas para a cultura do sorgo, com exceção dos bioestimulantes Stimulate ${ }^{\circledR}$ e Vorax ${ }^{\circledR}$, sendo relevante a realização de outros trabalhos que apliquem diferentes concentrações dos bioestimulantes nas sementes de sorgo granífero de modo a determinar uma dosagem que não prejudique a qualidade fisiológica das sementes da cultura do sorgo.

Vale ressaltar que os estudos a respeito dos efeitos de bioestimulantes, na germinação e vigor de sementes de sorgo granífero são escassos, especialmente dos produtos comerciais estudados aqui, uma vez, que as pesquisas que envolvem germinação da cultura estão mais voltados para nutrientes aplicados isoladamente, condições de estresse biótico e abiótico.

Os resultados obtidos para todos os caracteres comprovam que as dosagens técnicas dos bioestimulantes Vorax ${ }^{\circledR}$ e Zc Full Patriot ${ }^{\circledR}$ proporcionaram bons resultados para os caracteres de CP, PC e \%G, entretanto, os constituintes apresentaram toxicidade para as plântulas, sendo isto observado em relação ao caractere BSP, que resultou no menor acúmulo de biomassa seca, em contrapartida, o bioestimulante Stimulate ${ }^{\circledR}$ obteve bons resultados em todos os caracteres, em que, ou se igualou à testemunha, ou foi significativamente superior. A dosagem técnica do Awaken ${ }^{\circledR}$, por sua vez, foi prejudicial à qualidade fisiológica das sementes de sorgo granífero, fato comprovado pelos resultados obtidos para os caracteres PC, \%G e IVG.

Os resultados verificados por Mariucci et al. (2018) corroboram com os obtidos no presente estudo, uma vez que, verificaram que a aplicação da dosagem técnica do bioestimulante Awaken ${ }^{\circledR}$ em sementes de milho cultivar CD 324 PRO resultou em um maior efeito fitóxico em todos os tratamentos estudados, reduzindo drasticamente a qualidade fisiológica das sementes Em contrapartida, os mesmos autores infeririam que o bioestimulante Stimulate ${ }^{\circledR}$ não afetou de forma negativa a qualidade fisiológica das sementes de milho.

\section{Considerações Finais}

Sendo assim, conclui-se que o comprimento de plântula foi afetado positivamente por todos os bioestimulantes quando comparados com a testemunha. Além disso, pode-se inferir que o Awaken ${ }^{\circledR}$ conferiu $\circ$ menor desempenho perante os demais bioestimulantes usados, fato este, que é justificado pelos caracteres agronômicos de primeira contagem, porcentagem de germinação e índice de velocidade de germinação, por terem se apresentado inferiores à testemunha. Em relação à testemunha, tanto o bioestimulante Vorax ${ }^{\circledR}$ quanto $\circ$ Zc Full Patriot ${ }^{\circledR}$ tiveram resultados similares, com exceção da biomassa seca de plântula que foi inferior à da testemunha. Dentre os quatro bioestimulantes utilizados, o Stimulate ${ }^{\circledR}$ foi o que apresentou melhor resultado, pois, todas as características avaliadas foram iguais (primeira contagem, porcentagem de germinação e índice de velocidade de germinação) ou superiores (comprimento de plântula) às da testemunha.

\section{Agradecimentos}

À Universidade do Estado de Minas Gerais, Unidade Ituiutaba, pelo espaço para realização do estudo.

\section{Referências}

ALMEIDA FILHO, J. E. Avaliação agronômica e de estabilidade e adaptabilidade de híbridos de sorgo granífero. 2012.95 f. Dissertação (Mestrado) - Curso de Genética e Melhoramento de Plantas, Universidade Estadual do Norte Fluminense Darcy Ribeiro, Campos dos Goytcazes, 2012.

BONTEMPO, A. F.; ALVES, F. M.; CARNEIRO, G. D. O. P.; MACHADO, L. G.; SILVA, L. O. D.; AQUINO, L. A. Influência de bioestimulantes e nutrientes na emergência e no crescimento inicial de feijão, soja e milho. Revista Brasileira de Milho e Sorgo, v. I5, n, I, p.86-93, 20 I6. DOI: https://doi.org/I0.185 I2/I980-6477/rbms. vI5nIp86-93. Acesso em: 15 mar. 2019. 
BRASIL. Ministério da Agricultura, Pecuária e Abastecimento. Regras para análise de sementes. Ministério da Agricultura, Pecuária e Abastecimento. Secretaria de Defesa Agropecuária. - Brasília: Mapa/ACS, 2009. 399 p. Disponível em: https://www.abrates.org.br/files/regras_analise_de_sementes.pdf. Acesso em: 15 mar. 2019.

BUCHELT, A. C.; METZLER, C. R.; CASTIGLIONI, J. L.; DASSOLLER, T. F.; LUBIAN, M. S. Aplicação de bioestimulantes e Bacillus subtilis na germinação e desenvolvimento inicial da cultura do milho. Revista de Agricultura Neotropical, Cassilândia-MS, v. 6, n. 4, p. 69-74, out./dez. 2019. DOI: https://doi.org/I0.32404/rean.v6i4.2762. Acesso em: I5 mar. 2019.

COELHO, D. S.; SIMÕES, W. L.; MENDES, A. M. S.; DANTAS, B. F; RODRIGUES, J. A. S.; SOUZA, M. A. Germinação e crescimento inicial de variedades de sorgo forrageiro submetidas ao estresse salino. Revista Brasileira de Engenharia Agrícola e Ambiental, Campina Grande, v.18, n. I, p.25-30, Jan. 2014. DOl:https://doi.org/I0.1590/SI4I543662014000100004 .

COMPANHIA NACIONAL DE ABASTECIMENTO - CONAB. Acompanhamento da safra brasileira de grãos: décimo levantamento, julho 2020 - safra 2019/2020. Brasília: Companhia Nacional de Abastecimento. 2020.

CUNHA, S. G. S.; DAVID, A. M. S. S.; AMARO, H. T. R.; ALVES, D. D.; PORTO, E. M. V. Qualidade fisiológica de sementes de sorgo em resposta ao tratamento com fertilizante à base de zinco e molibdênio. Revista Agrarian, Dourados, v.8, n.30, p.35I-357, 20I5. Disponível em: http://ojs.ufgd.edu.br/index.php/agrarian/article/view/2979. Acesso em: 25 abr. 2020 .

CUNHA, S.P; SEVERO FILHO, W. A. Avanços tecnológicos na obtenção de etanol a partir de sorgo sacarino (Sorghum bicolor (L.) Moench). Revista Tecnológica, Santa Cruz do Sul-RS, v. 14, n. 2, p. 69-75, 2010. DOI: http://dx.doi. org/10.17058/tecnolog.v14i2.1523. Acesso em: 25 abr. 2020.

FERREIRA, L. L.; SOUZA, B. R.; PEREIRA, A. I. A.; CURVÊLO, C. R. S.; FERNANDES, C. S.; DIAS, N. S.; NASCIMENTO, E. K. A. Bioestimulante e nitrogênio de liberação gradual no desempenho do sorgo. Nativa, Sinop, v. 7, n. 4, p. 330-335, jul./ago. 2019. DOI: http://dx.doi.org/l0.3|4|3/nativa.v7i4.6656 Acesso em: 25 abr. 2020.

GASTL FILHO, J.; BONETTI, L. L. S.; ARAUJO, R. S.; DE SANTI, S.; NASCIMENTO, V. A.; VILARINHO, M. S. Salicylic acid and germinating potential in germination of cucumber seeds. Revista Inova Ciência \& Tecnologia, Uberaba, p.712, v.3, n.2, jul/dez., 2017. Disponível em: http://periodicos.iftm.edu.br/index.php/inova/article/view/257/229. Acesso em: 15 abr. 2020.

INOUE, M. H.; ARAÚJO, T. D. C.; MENDES, K. F.; BEN, R.; CONCIANI, P. A. Eficiência de dietholate e bioestimulante isolados e associados no tratamento de sementes de algodoeiro adensado com clomazone aplicado em pré-emergência. Revista de Ciências Agro-Ambientais, Alta Floresta-MT, v.10, n.2, p.163-172, 2012. Disponível em: http://www. unemat.br/revistas/rcaa/docs/voll0-2/4_modelo_artigo_rcaa_vlOn2a2012_miriam.pdf. Acesso em: 27 abr. 2020.

KOLLING, D. F.; SANGOI, L.; SOUZA, C. A.; SCHENATTO, D. E.; GIORDANI, W.; BONIATTI, C. M. Tratamento de sementes com bioestimulante ao milho submetido a diferentes variabilidades na distribuição espacial das plantas. Ciência Rural, Santa Maria, v.46, n.2, p.248-253, fev, 2016. DOI: http://dx.doi.org/10.1590/0 103-8478cr20150504. Acesso em: 27 abr. 2020.

LISBOA, L. A. M.; LAPAZ, A. M.; VIANA, R. S.; LEONEZI, R. S.; FIGUEIREDO, P. A. M. Influência do ácido salicílico no processo germinativo de sementes de cultivares de sorgo sacarino. Acta Iguazu, Cascavel, v.6, n.2, p. 37-49, 2017. Disponível em: http://e-revista.unioeste.br/index.php/actaiguazu/article/view/I 7446/I I633. Acesso em: 20 abr. 2020.

MAGUIRE, J. D. Speed of germination-aid in selection and evaluation for seedlig emergence and vigor. Crop Science, v. 2, n. I, p. I76-177, 1962. DOI: https://doi.org/I0.2135/cropscil 962.00III83X000200020033x. Acesso em: 27 abr. 2020.

MARIUCCI, G. E. G.; SUZUKAWA, A. K.; BRACCINI, A. L; SCAPIM, C. A.; LIMA, L. H. S.; ANGELOTTI, P.; PONCE, R. M.; MARTELI, D. C. V. Physiological potential of maize seeds submitted to different treatments and storage periods. Journal of Seed Science, Londrina, v.40, n. I, p.60-66, Mar. 20 I8. DOI: https://doi.org/I0.1590/23 I7-I545v40nI I84456. Acesso em: 27 abr. 2020.

NASSIF, S. M. L.; PEREZ, S. C. J. G. Efeito da temperatura na germinação de sementes de Amendoim-do-campo (Pterogyne nitens Tul.). Revista Brasileira de Sementes, Brasília, DF, v. 22, n. I, p. I-6, 2000.

PARRELLA, R. A. C.; RODRIGUES, J. A. S.; TARDIN, F. D. SCHAFFERT, R. E. Desenvolvimento de Híbridos de Sorgo Sensíveis ao Fotoperíodo visando Alta Produtividade de Biomassa. Sete Lagoas: Embrapa Milho e Sorgo, 2010. 25 p. (Embrapa Milho e Sorgo. Boletim de Pesquisa e Desenvolvimento, 28). 
R CORE TEAM. R: A language and environment for statistical computing. R Foundation for Statistical Computing, Vienna, Austria. 2019.

ROCHA, A. G. C. Adensamento de plantas na cultura do sorgo granífero. 20I7. 48 f. Dissertação (Mestrado) Curso de Produção Vegetal, Universidade de Rio Verde, Rio Verde, 2017.

RODRIGUES, L. R.; GUADAGNIN, J. P.; PORTO, M. P. Indicações técnicas para o cultivo de milho e de sorgo no Rio Grande do Sul - Safras 2009/201 0 e 201 0/20I I.Veranópolis: FEPAGRO - Serra, 2009. 179 p.

SANTOS, C. A. C.; PEIXOTO, C. P.; VIEIRA, E. L., CARVALHO, E. V.; PEIXOTO, V. A. B. Stimulate ${ }^{\circledR}$ na germinação de sementes, emergência e vigor de plântulas de girassol. Bioscience Journal, Uberlândia, v. 29, n. 2, p. 605-6I6, 20I3. Disponível em: http://www.seer.ufu.br/index.php/biosciencejournal/article/ view/l409 I/I 2502. Acesso em: 19 abr. 2020.

SANTOS, G. A. Composição química e características fermentativas de silagem de erva sal aditivas com concentrados energéticos. 20 I l. 64 f. Dissertação (Mestrado) - Curso de Ciência Animal, Universidade Federal do Vale do São Francisco, Petrolina, $201 \mathrm{I}$.

SILVA, T. T. A.; VON PINHO, E. V. R; CARDOSO, D. L.; FERREIRA, C. A.; ALVIM, P. O.; COSTA, A. A. F. Qualidade fisiológica de sementes de milho na presença de bioestimulantes. Ciência e Agrotecnologia, v.32, n.3, p.840-846, 2008. DOI: https://doi.org/I0.I590/SI4 I3-7054200800030002I. Acesso em: I 9 abr. 2020.

TATTO, L.; KULCZYNSKY, S. M.; BELLÉ, C.; MORIN, D.; RUBIN, F. M. ULIANA, M. P. Desempenho de sementes de soja tratadas com bioestimulante sob diferentes condições de potencial osmótico. Revista Eletrônica Científica da EURGS, v.4, n.3, p.397-408, 20 I8. DOI: http://dx.doi.org/I0.2 I674/2448-0479.43.397-408. Acesso em: 19 abr. 2020.

VAZQUEZ, G. H.; BERTOLIN, D. C.; SPEGIORIN, C. N. Testes de envelhecimento acelerado e de condutividade elétrica para avaliar a qualidade fisiológica de sementes de sorgo (Sorghum bicolor (L.) Moench). Revista Brasileira de Biociências, Porto Alegre, v. 9, n. I, p. I8-24, jan./mar. 20I I. Disponível em: http://www.ufrgs.br/seerbio/ojs/index. php/rbb/article/view/I 504. Acesso em: 20 abr. 2020.

VENDRUSCOLO, E. P.; SANTOS, O. F; ALVES, C. Z. Substâncias húmicas na qualidade fisiológica de sementes de sorgo. Journal of Agronomic Sciences, Umuarama, v.3, n.2, p. I69-I77, 2014.

WORMA, M.; SEGATTO, C.; STEFEN, D.; BUBA, G. P.; LEOLATO, L. S. Qualidade fisiológica de sementes de milho produzidas com adubação biológica e bioestimulante em diferentes preparos de solo. Engenharia na Agricultura, v.27, n.3, p. 187-194, 2019. DOI: http://dx.doi.org/I0.13083/reveng.v27i3.893. Acesso em: 20 abr. 2020. 